\title{
Appetite is not a reliable predictor of energy intake: interim reporting of a systematic literature review
}

\author{
S. Till ${ }^{1}$, C. J. Harden ${ }^{1,3}$, V. A. Grant ${ }^{1,2}$ and B. M. Corfe ${ }^{1}$ \\ ${ }^{1}$ Molecular Gastroenterology Research Group, University of Sheffield, ${ }^{2}$ Department of English, University of Sheffield \\ and ${ }^{3}$ Research and Innovation Services, University of Sheffield, S10 $2 T N$
}

Subjective ratings of appetite, derived from visual analogue scales, are widely used stock-in-trade in appetitive research. These instruments follow a general format and may include a series of decoy questions, closely related questions (such as on motivation to eat and fullness), and scale reversals ${ }^{(1)}$. They are applied in studies that are designed to assess whether interventions have the potential to modify appetite, with the implicit or explicit inference that this will in turn modify energy intake (EI). Our group noted in previous studies that appetitive scores did not relate strongly to actual scores of subsequent EI. An informal survey of the literature suggested this disconnect was common in other papers, but also unremarked ${ }^{(2,3)}$. We therefore aim to undertake a systematic review of literature (SLR) reporting both rated appetite and EI to establish whether subjective ratings of appetite are reliable predictors of energy intake.

In line with guidance on SLR, the searches have been undertaken by two independent workers using the agreed sets of terms and using three independent databases (Scopus, Medline, Web of Knowledge). Endnote is then used to de-duplicate lists from databases. The identified papers are manually screened for relevance: reviews, non-human and other non-relevant papers are excluded, yielding a set for analysis. The final list of papers will then be assessed by two researchers independently and scored with a binary outcome as to whether a link does or does not exist. At point of reporting, a screen from 2003-2014 de duplication and triage has yielded 582 papers for analysis. 442 have so far been included in the analysis. Only a small subset of papers $n=9$, report a direct comparison of rated appetite and actual intake, so the majority of outcomes are inferential. Of 295 papers found to be relevant, $81 \%$ showed no relationship between rated appetite and energy intake. Of those with a direct comparison $56 \%$ showed no relationship between rated appetite and energy intake.

(a)

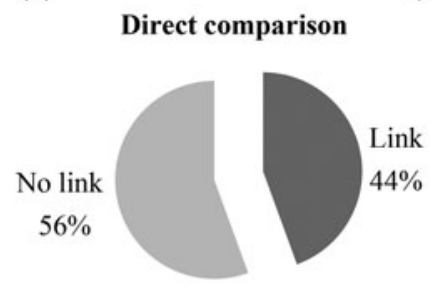

(b)

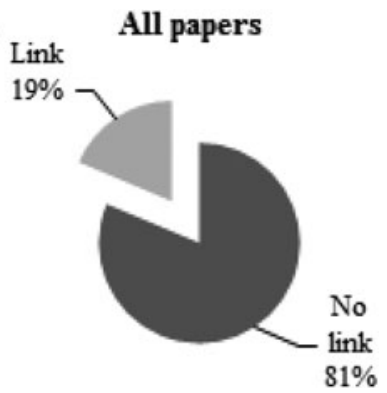

Fig. 1. Proportions of papers show link or otherwise between appetite ratings and energy intake. Panel A summarises papers directly comparing the endpoints $(n=9)$. Panel B summarises all papers reporting both endpoints $(n=276)$

Whilst this report is incomplete, fewer than half of the 442 papers assessed showed a relationship between rated appetite and energy intake. If the trend holds throughout the SLR, the analysis suggests that appetite is not a reliable predictor of EI. Future work will use text mining approaches and appreciative inquiry to explore the possibility of a "gold standard" experimental design.

1. Blundell J et al. (2010) Appetite Control: Methodological aspects of the evaluation of food. Obes Rev 11(3), 251-270.

2. Alfenas R. Mattes (2003) R. Effect of fat sources on satiety. Obes Res 11(2), 183-187.

3. Majjars J et al. (2009) Effect ofs fat saturation on satiety, hormone release and food intake. Am J Clin Nutr 89(4), 1019-1024. 